\title{
Leucrose, a Sucrose Isomer, Suppresses Hepatic Fat Accumulation by Regulating Hepatic Diet-induced Obese Mice
}

\author{
Jihye Lee ${ }^{1}$, Eunju Kim ${ }^{1}$, Yuri Kim ${ }^{1}$, Sang-Ho Yoo ${ }^{2}$ \\ ${ }^{1}$ Department of Nutritional Science and Food Management, Ewha Womans University, ${ }^{2}$ Department of Food Science and Biotechnology, and \\ Carbohydrate Bioproduct Research Center, Sejong University, Seoul, Korea
}

\begin{abstract}
Obesity is currently one of the most serious public health problems and it can lead to numerous metabolic diseases. Leucrose, D-glucopyranosyl- $\alpha$-(1-5)-D-fructopyranose, is an isoform of sucrose and it is naturally found in pollen and honey. The aim of this study was to investigate the effect of leucrose on metabolic changes induced by a high-fat diet (HFD) that lead to obesity. C57BL/6 mice were fed a $60 \%$ HFD or a HFD with $25 \%$ (L25) or $50 \%$ (L50) of its total sucrose content replaced with leucrose for 12 weeks. Leucrose supplementation improved fasting blood glucose levels and hepatic triglyceride content. In addition, leucrose supplementation reduced mRNA levels of lipogenesis-related genes, including peroxisome proliferator-activated receptor $\gamma$, sterol regulatory element binding protein 1C, and fatty acid synthase in HFD mice. Conversely, mRNA levels of $\beta$ oxidation-related genes, such as carnitine palmitoyltransferase $1 \mathrm{~A}$ and acyl COA oxidase, returned to control levels with leucrose supplementation. Taken together, these results demonstrated the therapeutic potential of leucrose to prevent metabolic abnormalities by mediating regulation of plasma glucose level and hepatic triglyceride accumulation.
\end{abstract}

(J Cancer Prev 2018;23:99-106)

Key Words: Leucrose, High fat diet, Lipogenesis, Liver

\section{INTRODUCTION}

Obesity is currently the most concerning health problem worldwide due to its contribution to the development of chronic diseases, such as type 2 diabetes mellitus (T2DM), insulin resistance, cardiovascular disease, non-alcoholic fatty liver disease (NAFLD), and dyslipidemia. ${ }^{1,2}$ In NAFLD, more than $5 \%$ of liver volume is infiltrated by triglycerides (TGs) in the absence of alcohol intake, and this leads to an imbalance in lipid storage and excretion. ${ }^{3}$ Under normal physiological conditions, the liver contains low concentrations of TG. In NAFLD, liver can accumulate TG via uptake of free fatty acids from plasma and from de-novo fatty acid synthesis and lipogenesis. The latter two processes are primarily regulated at the transcriptional level. ${ }^{3}$ However, an imbalance between lipid acquisition from these two sources and their removal (via $\beta$-oxidation and export as very low density lipoproteins) can lead to an accumulation of TG in the liver. ${ }^{4}$

Sterol regulatory element binding protein 1C (SREBP-1C) is an essential membrane-bound transcription factor that contributes to hepatic fatty acid synthesis by activating lipogenic genes. ${ }^{5}$ It has been reported that SREBP-1C plays a role in the accumulation

Received June 12, 2018, Revised June 21, 2018, Accepted June 24, 2018

Correspondence to: Yuri Kim

Department of Nutritional Science and Food Management, Ewha Womans University, 52 Ewhayeodae-gil, Seodaemun-gu, Seoul 03760, Korea

Tel: +82-2-3277-4485, Fax: +82-2-3277-2862, E-mail: yuri.kim@ewha.ac.kr, ORCID: Yuri Kim, https://orcid.org/0000-0001-7606-8501

Correspondence to: Sang-Ho Yoo

Department of Food Science and Biotechnology, and Carbohydrate Bioproduct Research Center, Sejong University, 209 Neungdong-ro, Gwangin-gu, Seoul 05006, Korea

Tel: +82-2-3408-3221, Fax: +82-2-3408-4319, E-mail: shyoo@sejong.ac.kr, ORCID: Sang-Ho Yoo, https://orcid.org/0000-0002-7258-5090

Copyright (C) 2018 Korean Society of Cancer Prevention

(c) This is an Open Access article distributed under the terms of the Creative Commons Attribution Non-Commercial License (http://creativecommons.org/icenses/by-n//4.0) which permits unrestricted non-commercial use, distribution, and reproduction in any medium, provided the original work is properly cited. 
of TG in the liver by regulating fatty acid synthase and acetyl Co-A carboxylase $1 .^{5.6}$ PPAR $\gamma$, a nuclear hormone receptor, is also involved in the pathophysiology of fatty liver by mediating up-regulation of $C D 36$, a downstream target of PPAR $\gamma$ in high-fat diet (HFD)- induced mice. ${ }^{7}$ Hepatic steatosis induced by HFD in mice was found to be improved when hepatocyte-specific deletion of PPAR $\gamma$ was achieved, and this was accompanied by a down-regulation of genes involved in lipogenesis and $\beta$-oxidation. ${ }^{8}$ PPAR $\alpha$ is also major regulator to maintain the balance of liver TG by activating $\beta$-oxidation genes. ${ }^{9}$ Carnitine palmitoyltransferase $1 \mathrm{~A}$ and acyl $\mathrm{CoA}$ oxidase contribute to $\beta$-oxidation in the liver and they both have the major roles in maintaining the hepatic balance of TG. ${ }^{10,11}$

In recent years, the increased intake of sugar-sweetened beverages and food has been shown to predispose individuals to metabolic diseases, including NAFLD. ${ }^{12,13}$ Due to the rapid absorption of sugar in the small intestine, sugar represents a quick energy source. However, high consumption of sugar can lead to excess weight gain, T2DM, cardiovascular disease, and liver fat accumulation. ${ }^{14}$ In more recent years, zero calorie and low-calorie sweeteners have been developed to prevent the high intake of sugar. However, the sweeteners are associated with side effects, such as gastrointestinal discomfort and increased glucose intolerance. ${ }^{15}$

There are several sucrose isoforms, including isomaltulose (palatinose), trehalulose, maltulose, turanose, and leucrose. ${ }^{16}$ Isomaltulose is characterized by a reduced digestive rate in the small intestine compared with sucrose and an ability to slowly increase blood glucose level and reduce visceral fat accumulation. ${ }^{16,17}$ Meanwhile, turanose has been shown to down-regulate adipogenesis-related gene expression in 3T3L1 adipocytes and to suppress inflammatory cytokines in Raw 264.7 macrophages. ${ }^{18,19}$

Leucrose, D-glucopyranosyl- $\alpha$-(1-5)-D-fructopyranose, naturally exists in pollen and honey and was first discovered in studies of the fermentation process of dextran from sucrose in the bacteria, Leuconostoc mesenteroides. ${ }^{20.21}$ Leucrose is hydrolyzed into glucose and fructose by $\alpha$-glucosidase in the small intestine and its rate of hydrolyzation is slower than that of sucrose. ${ }^{22}$ Recently, it was reported that leucrose inhibited intracellular lipid accumulation and regulated adipogenic genes in 3T3-L1 adipocytes. ${ }^{23}$ Therefore, in this study, we investigated whether leucrose supplementation could affect obesity-related lipid accumulation in the liver of HFD-induced obese mice.

\section{MATERIALS AND METHODS}

\section{Materials}

Leucrose was purchased from Carbosynth Limited (Berkshire, UK) and kept in an auto-desiccator (Sanpla Dry Keeper; Sanplatec Corp, Osaka, Japan).

\section{Animals and diet}

Five-week-old male C57BL/6 mice (18-20 g) were purchased from Central Lab Animal Inc. (Seoul, Korea) and adapted to controlled conditions $\left(22^{\circ} \mathrm{C} \pm 2^{\circ} \mathrm{C}, 50 \% \pm 5 \%\right.$ humidity, 12 -hour light-dark cycle) for one week. The mice were provided American Institute of Nutrition 93G (AIN93G) diet and water ad libitum. After one week, total of 40 mice were randomized into four groups as follows: (a) a control group ( $\mathrm{Ctrl}, \mathrm{n}=10$ ) which received AIN93G diet; (b) a HFD-induced obese control group (HF, $n=10$ ) which received a HFD ( $60 \%$ of total calories from fat); (c) a leucrose $25 \%$ group (L25, $\mathrm{n}=10$ ) which received a HFD with $25 \%$ of its total sucrose content replaced with leucrose, and (d) a leucrose 50\% group (L50, $\mathrm{n}=10$ ) which received a HFD with 50\% of its total sucrose content replaced with leucrose. Experiments diets were purchased from Unifaith (Seoul, Korea) and provided for 12 weeks before sacrifice. Diet intake and body weight were monitored twice a week. The experimental protocol for this study was approved by the Institutional Animal Care and Use Committee of Ewha Womans University (IACUC approval no: 17-021).

\section{Biochemical analysis of blood samples}

Fasting blood glucose was measured prior to sacrifice of the mice. Briefly, after 12 hours of fasting, blood samples were collected from the tail vein and were analyzed by using a portable glucometer (Roche Diagnostics GmbH, Mannheim, Germany). Next, the mice were anesthetized with isoflurane (Piramal Critical Care, Bethlehem, PA, USA) for collection of whole blood from the inferior vena cava. The samples were immediately centrifugated at $3,000 \mathrm{rpm}$ at $4^{\circ} \mathrm{C}$ for 15 minutes to obtain serum. The serum samples were immediately stored at $-80^{\circ} \mathrm{C}$. Serum levels of TG, total cholesterol (TC), and high-density lipoprotein cholesterol (HDL-C) were measured with a commercially available kit (Asan Pharmaceutical Co., Seoul, Korea). Low-density lipoprotein cholesterol (LDL-C) levels were calculated as follows: $\mathrm{LDL}-\mathrm{C}=\mathrm{TC}-\mathrm{HDL}-\mathrm{C}-(\mathrm{TG} / 5)$. Serum levels of insulin were determined with a commercially available ELISA kit (Crystal; Downers Grove, IL, USA). 


\section{Histological and triglyceride analysis in liver tissues}

For H\&E staining, liver tissues were fixed in $10 \%$ formaldehyde and then embedded in paraffin wax. The paraffin blocks were sectioned (3-4 $\mu \mathrm{m} /$ slice) and stained with H\&E followed by deparaffinized with xylene. Stained sections were photographed with a Nikon microscope (Nikon, Tokyo, Japan) at 100× magnification.

Concentration of hepatic was analyzed as previously described. ${ }^{24}$ Briefly, frozen liver tissues (100 $\pm 20 \mathrm{mg}$ ) were incubated with ethanolic potassium hydroxide solution $(\mathrm{KOH})(1: 2 \mathrm{v} / \mathrm{v}$ of $30 \%$ $\mathrm{KOH}$ and $100 \%$ ethanol) at $55^{\circ} \mathrm{C}$ overnight. A solution of $50 \%$ ethanol was then added prior to centrifugation of the decomposed tissues at $8,000 \mathrm{rpm}$ for 5 minutes. The obtained supernatant was incubated on ice for 10 minutes after addition of $50 \%$ ethanol and $1 \mathrm{M} \mathrm{MgCl}_{2}$. And the TG content was measured by TG reagent, free glycerol reagent (Sigma-Aldrich, St. Louis, MO, USA) after centrifugation at $8,000 \mathrm{rpm}$ for 5 minutes. Quantification was done by comparing to glycerol standard curve.

\section{RNA extraction and reverse transcription-PCR}

Total RNA was isolated by homogenizing liver tissues in TRIzol reagent (Invitrogen, Carlsbad, CA, USA). Concentration and integrity of each total RNA sample was measured with a NanoDrop 2000 system (Thermo Scientific, Wilmington, DE, USA) and cDNA was synthesized from $2 \mu$ of each RNA samples with a RevertAid First Strand cDNA synthesis kit (Thermo Fisher Scientific). Amplification of cDNA was performed with Taq polymerase (TAKARA, Tokyo, Japan) and the conditions of amplification were as follows: an initial incubation at $94^{\circ} \mathrm{C}$ for 5 minutes was followed by denaturation at $94^{\circ} \mathrm{C}$ for 30 seconds, an annealing step at $56^{\circ} \mathrm{C}$ for 30 seconds, and an extension step at $72^{\circ} \mathrm{C}$ for 2 minutes. The resulting PCR products were separated in $2 \%$ agarose gels by electrophoresis and visualized with UV light. The primers are as follows: mouse Acox1, 5'-TTGGAAACCAC TGCCACATA-3' (forward) and 5'-AGGCATGTAACCCGTAGCAC-3' (reverse); mouse Cptla, 5'AACCCAGTGCCTTAACGATG-3' (forward) and 5'-GAACTGGTGGCCAATGAGAT-3' (reverse); mouse Fasn, 5'-TGTGAGTGGTTCAGAGGCAT-3' (forward) and 5'TTCTGTAGTGCC AGCAAGCT-3' (reverse); mouse Ppara, 5'-CCAA CATGGTGGACACAGAG-3' (forward) and 5'-GCAAAACCAAAGCT TCCAGA-3' (reverse); mouse Pparg, 5'-GAGCACTTCACA AGAA ATTACC-3' (forward) and 5'-GAACTCCATAGTGGAAGCCT-3' (reverse); mouse Srebf1, 5'-TAGAGCATATCCCCCAGGTG-3' (forward) and 5'-GGTACGGGCCACAAGAAGTA-3' (reverse); mouse Actb, 5'CGCCACCAGTTCGCCATGGA-3' (forward) and 5'-TACAGCCCGG GGAGCATCGT-3' (reverse). The relative expression of mRNA was normalized to a house keeping gene, $\beta$-actin. And all PCR results were analyzed from 10 mice from each group and the PCR blots were quantified using Image J software (National Institutes of Health, USA).

\section{Statistical analysis}

Data were analyzed with GraphPad Prism (GraphPad Software, Inc., La Jolla, CA, USA) and presented as the mean \pm SEM. Data were analyzed by one-way ANOVA followed by Newman-Keuls's post-hoc test. A $P$-value less than 0.05 was considered to indicate statistical significance.

Table 1. Body weight, liver weight, levels of fasting blood glucose, serum insulin, and serum lipid profiles after 12 weeks of experiment

\begin{tabular}{lrrrr}
\hline \multicolumn{1}{c}{ Variable } & \multicolumn{1}{c}{ Ctrl } & \multicolumn{1}{c}{ HF } & \multicolumn{1}{c}{ L25 } & L50 \\
\hline Final body weight $(\mathrm{g})$ & $38.11 \pm 0.66^{\mathrm{a}}$ & $44.43 \pm 1.13^{\mathrm{b}}$ & $44.43 \pm 0.81^{\mathrm{b}}$ & $44.31 \pm 0.80^{\mathrm{b}}$ \\
Liver tissue weight (g) & $1.48 \pm 0.06^{\mathrm{a}}$ & $2.39 \pm 0.16^{\mathrm{b}}$ & $2.15 \pm 0.16^{\mathrm{b}}$ & $2.06 \pm 0.16^{\mathrm{b}}$ \\
Liver/body weight ratio (\%) & $3.87 \pm 0.11^{\mathrm{a}}$ & $5.34 \pm 0.25^{\mathrm{b}}$ & $4.81 \pm 0.27^{\mathrm{b}}$ & $4.62 \pm 0.28^{\mathrm{b}}$ \\
Fasting blood glucose (mg/dL) & $108.50 \pm 3.24^{\mathrm{a}}$ & $184.80 \pm 6.72^{\mathrm{b}}$ & $141.10 \pm 11.47^{\mathrm{c}}$ & $133.20 \pm 7.48^{\mathrm{c}}$ \\
Serum insulin (ng/mL) & $0.86 \pm 0.09^{\mathrm{a}}$ & $2.45 \pm 0.21^{\mathrm{b}}$ & $2.73 \pm 0.28^{\mathrm{b}}$ & $2.50 \pm 0.25^{\mathrm{b}}$ \\
TG (mg/dL) & $122.62 \pm 2.56^{\mathrm{a}}$ & $138.73 \pm 4.56^{\mathrm{b}}$ & $135.59 \pm 3.82^{\mathrm{b}}$ & $127.99 \pm 2.54^{\mathrm{b}}$ \\
TC (mg/dL) & $120.37 \pm 3.41^{\mathrm{a}}$ & $157.87 \pm 3.74^{\mathrm{b}}$ & $161.40 \pm 6.75^{\mathrm{b}}$ & $151.87 \pm 5.30^{\mathrm{b}}$ \\
HDL-cholesterol (mg/dL) & $85.97 \pm 1.71^{\mathrm{a}}$ & $73.19 \pm 0.93^{\mathrm{b}}$ & $69.95 \pm 2.01^{\mathrm{b}}$ & $59.58 \pm 1.51^{\mathrm{c}}$ \\
LDL-cholesterol (mg/dL) & $9.88 \pm 3.04^{\mathrm{a}}$ & $56.93 \pm 3.70^{\mathrm{b}}$ & $64.33 \pm 7.96^{\mathrm{b}}$ & $66.69 \pm 5.61^{\mathrm{b}}$ \\
\hline
\end{tabular}

Values are presented as mean \pm SEM. The different superscript of $a, b$, and $c$ indicate significant differences. Ctrl, control mice that received the AIN93G diet; HF, obesity control mice that received a high-fat diet (HFD); L25, mice that received a HFD with $25 \%$ of the total sucrose replaced with leucrose; L50, mice that received a HFD with 50\% of the total sucrose replaced with leucrose; TG, triglyceride; TC, total cholesterol; HDL, high-density lipoprotein; LDL, low-density lipoprotein. 


\section{RESULTS}

\section{Effect of leucrose on body weight, liver weight, fasting blood glucose level, and blood lipid profiles}

After 12 weeks of receiving HFD, the HF group exhibited a significant increase in body weight compared to the $\mathrm{Ctrl}$ group $(P<$ 0.001 ). However, body weights did not significantly differ between the HF and leucrose supplementation groups (Table 1). While leucrose supplementation also did not affect adipose tissue weight (data not shown), absolute liver tissue weight and relative liver weight to body weight tended to be lower with leucrose supplementation, although differences were not significant.

The HF group exhibited a significant increase in fasting blood glucose level compared to the Ctrl group $(P<0.001)$, while, fasting blood glucose levels were lower in both the L25 and L50 groups compared to the HF group ( $P<0.001$ for both). In contrast, serum levels of insulin were not affected by leucrose supplementation. Serum levels of TG, TC, and LDL-cholesterol were significantly higher in the $\mathrm{HF}$ group compared to the $\mathrm{Ctrl}$ group ( $P<0.05$ for TG, $P<0.001$ for TC and LDL-cholesterol). Furthermore, levels of TG tended to be lower in the L50 group, although the decrease was not statistically significant. The serum level of HDL-cholesterol was significantly lower in the HF group and in both the L25 and L50 groups (all $P<0.001$ ).

\section{Effect of leucrose on histological changes and concentrations of triglyceride in liver tissue}

H\&E staining of liver tissues revealed an increase in hepatic lipid droplet accumulation in the HF group compared with the Ctrl group (Fig. 1A). In contrast, liver tissue from the L25 and L50 groups contained fewer lipid droplets (Fig. 1A). Consistent with this result, a significant increase in levels of TG was detected in liver tissues from the HF group, while the levels of TG was reduced in the $\mathrm{L} 50$ group $(P<0.01)$ (Fig. 1B).

\section{Effect of leucrose on lipogenesis-related gene expression in liver tissue}

To verify the effect of leucrose on lipogenesis in the liver, mRNA levels of Pparg, Srebf1, and Fasn were analyzed (Fig. 2). All three mRNA levels were higher in the HF group compared to the Ctrl group ( $P<0.001$ for Pparg, Srebf1 $P<0.05$ for Fasn), while all three levels were down-regulated in the L25 and L50 groups. In particular, the mRNA expression of Pparg was significantly down-regulated in the L25 and L50 groups compared to the HF group ( $P<0.01$ and $P<0.001$, respectively), and the mRNA expression of Srebf 1 was down-regulated in both leucrose supplementation groups compared to the HF group $(P<0.05$ for all) (Fig. 2B-2C). Leucrose supplementation also tended to down-regulate expression of Fasn, although the effect was not significant (Fig. 2D).
A

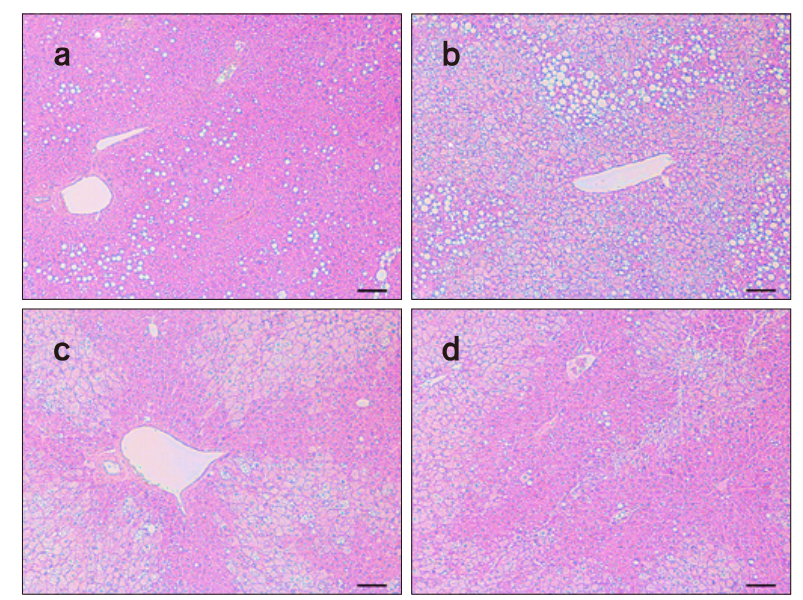

B

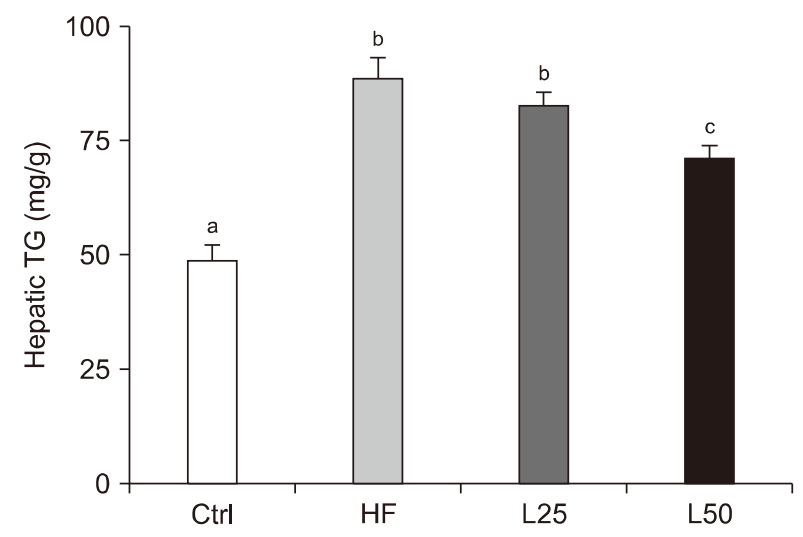

Figure 1. Leucrose supplementation suppressed high-fat diet (HFD)-induced triglyceride (TG) accumulation in liver. (A) H\&E staining of liver tissues from (a) Ctrl, (b) HF, (c) L25, and (d) L50 groups. Bar, $50 \mu \mathrm{m}$. (B) TG content of liver tissues (mg/g). Data are represented as the mean \pm SEM. The different letters of $a, b$, and $c$ on the bar graph indicate significant differences. Ctrl, control mice which received the AIN-93G diet; HF, mice which received a HFD to induce obesity; L25, mice which received a HFD with $25 \%$ of its total sucrose content replaced with leucrose; L50, mice which received a HFD with 50\% of its total sucrose content replaced with leucrose. 
A
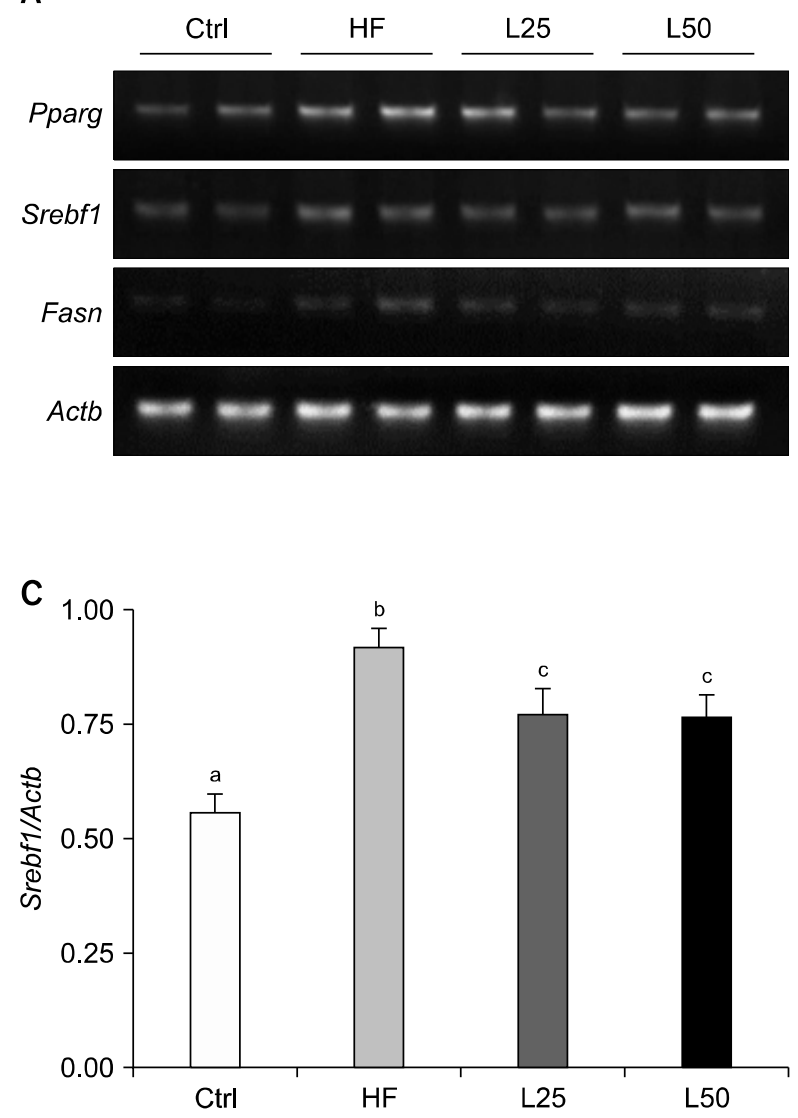

B

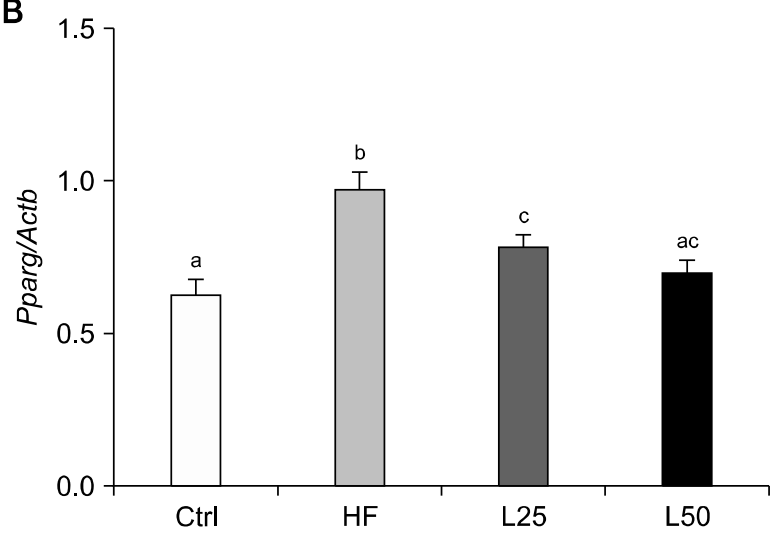

D

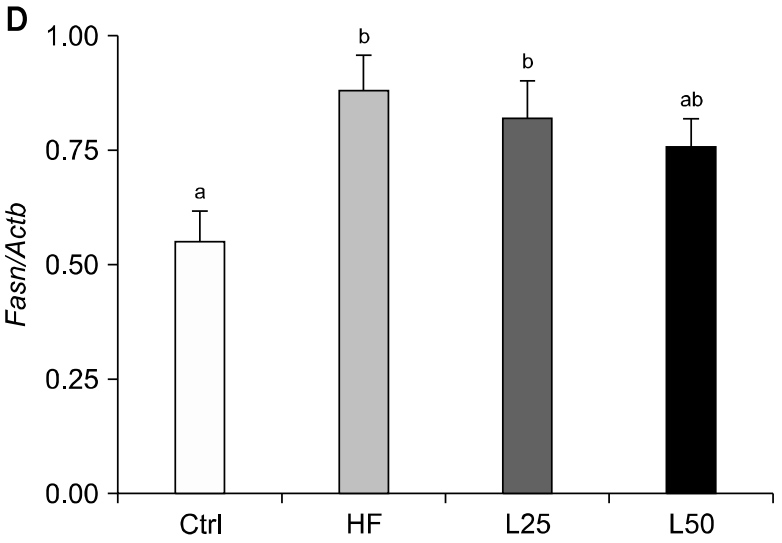

Figure 2. Leucrose supplementation regulated adipogenesis-related gene expression in liver. Representative blots (A), quantified expression levels of Pparg (B), Srebf1 (C), and Fasn (D). Actb was used as a loading control. Data are represented as the mean \pm SEM. The different letters of $a, b$, and $c$ on the bar graph indicate significant differences. Ctrl, control mice which received the AIN-93G diet; HF, mice which received a HFD to induce obesity; L25, mice which received a HFD with 25\% of its total sucrose content replaced with leucrose; L50, mice which received a HFD with $50 \%$ of its total sucrose content replaced with leucrose; Srebf1, sterol regulatory element-binding protein 1c; Fasn, fatty acid synthase.

\section{Effect of leucrose on $\beta$-oxidation-related gene expressions in liver tissue}

To determine whether leucrose affects $\beta$-oxidation in the liver, mRNA levels of Ppara, Cpt1a, and Acox1 were analyzed (Fig. 3A). For Ppara and Acox1, their mRNA levels in the L50 group were significantly higher compared to the HF group $(P<0.05)$ (Fig. 3B and 3D). Cptla mRNA was significantly down-regulated in the $\mathrm{HF}$ group compared to the Ctrl group ( $P<0.001$ ) (Fig. 3C). In contrast, mRNA levels of Cptlawere up-regulated in both the L25 and L50 groups (all $P<0.05$ ).

\section{DISCUSSION}

In this study, we demonstrated that leucrose, a natural sucrose isomer, suppresses obesity-related hepatic lipid accumulation by regulating hepatic lipogenesis and $\beta$-oxidation in HFD-induced obese mice. In particular, leucrose improved the levels of fasting blood glucose and hepatic TG. Molecular analyses of liver tissues further demonstrated that leucrose supplementation downregulated genes associated with lipogenesis and up-regulated genes associated with $\beta$-oxidation.

Dietary consumption of sugar represents intake of a high energy resource. However, in excess, intake of sugar can lead to obesity and related metabolic diseases, including NAFLD and hepatic steatosis. Furthermore, when diets are high in sucrose and fat, serum and hepatic levels of TG are increased. ${ }^{25}$ Recently, the potential for various sweeteners to serve as alternatives to sugar to reduce blood glucose levels and fat deposition in obese models was examined. ${ }^{26}$ Leucrose is produced following the isomerization of sucrose molecules by dextransucrase and it possesses half the sweetness of sucrose. ${ }^{21}$ However, the 
A
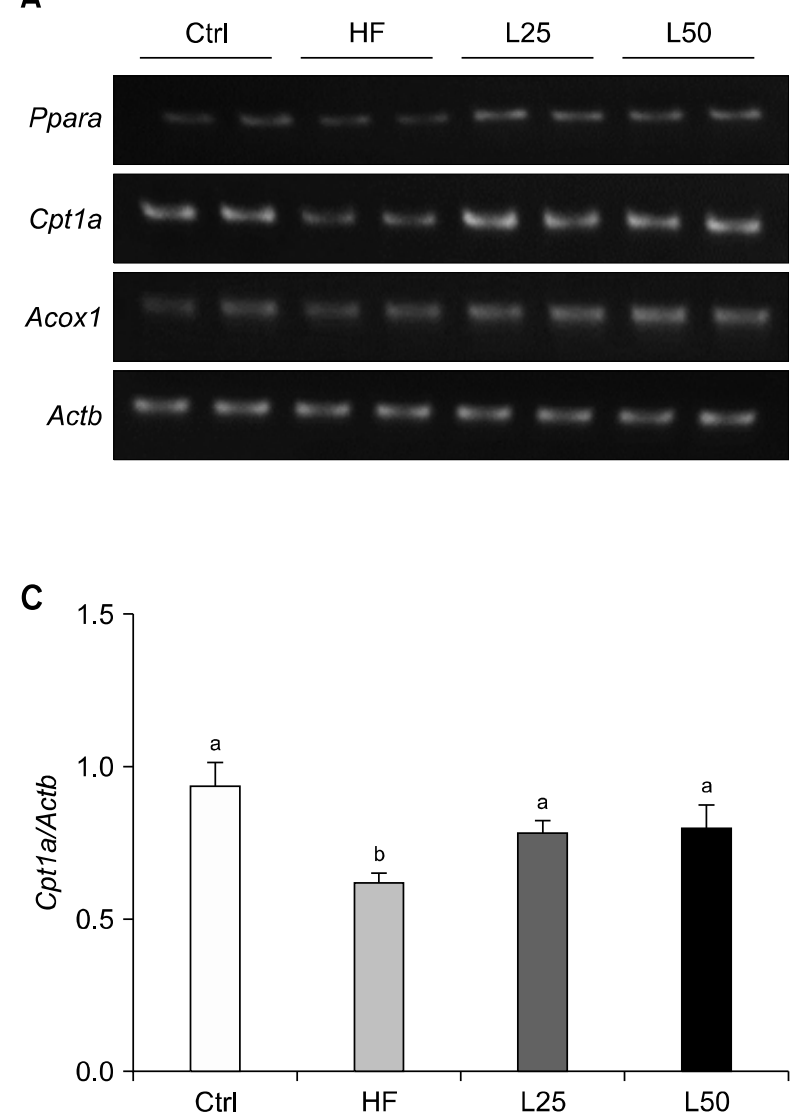

B

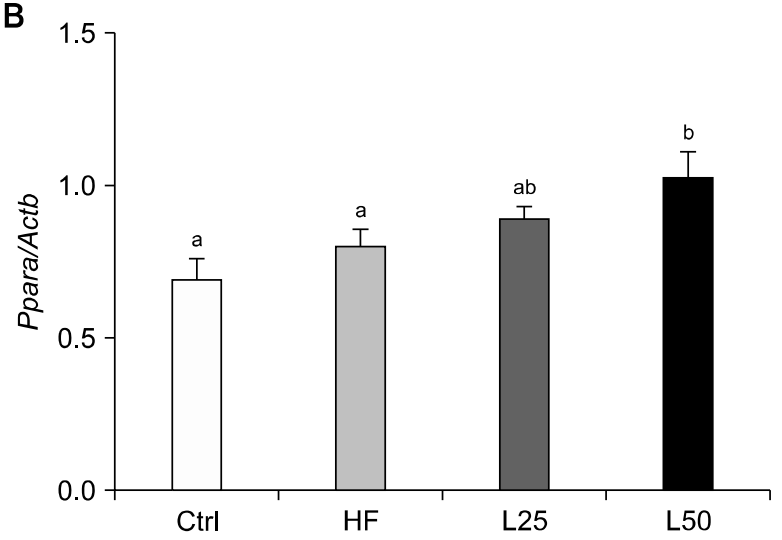

D

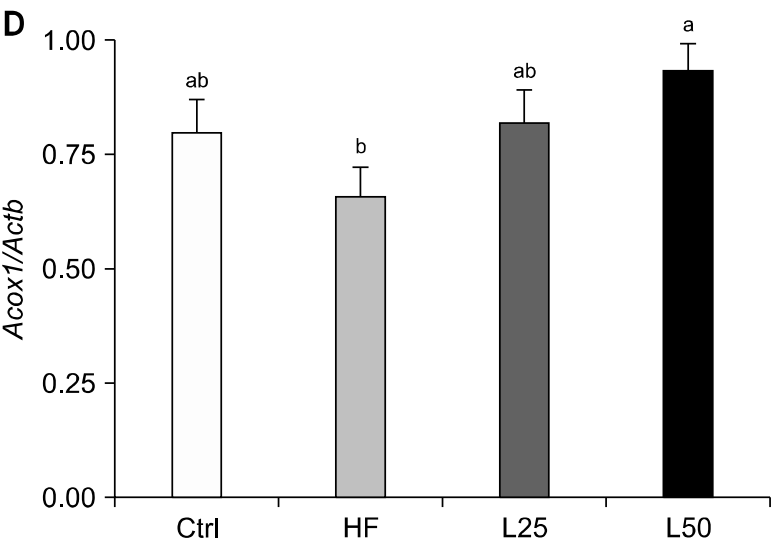

Figure 3. Leucrose supplementation regulated the $\beta$-oxidation-related gene expression in liver. (A) Representative blots, quantified expression levels of Ppara (B), Cpt1a (C), and Acox1 (D). Actb was used as a loading control. Data are represented as the mean \pm SEM. The different letters of $\mathrm{a}$ and $\mathrm{b}$ on the bar graph indicate significant differences. Ctrl, control mice which received the AIN-93G diet; HF, mice which received a HFD to induce obesity; L25, mice which received a HFD with $25 \%$ of its total sucrose content replaced with leucrose; L50, mice which received a HFD with 50\% of its total sucrose content replaced with leucrose; Cpt1a, carnitine palmitoyltransferase 1A; Acox1, acyl-CoA oxidase.

physiological effects of leucrose have not been extensively studied. It has been reported that $50 \%, 75 \%$, or $100 \%$ replacement of glucose with leucrose can suppress adipogenesis by down-regulating genes related to adipogenesis in 3 T3 L1 adipocytes. ${ }^{23}$ However, in the present study, no significant differences in body weight or adipocyte mass were detected when $25 \%$ or $50 \%$ of dietary sucrose was replaced with leucrose. These distinct results may be due to differences in replacement for glucose in vitro cell study and for sucrose in vivo animal study.

When $100 \%$ of sucrose was replaced with isomaltulose, no significant changes in body weight or energy intake were observed compared with a sucrose diet group. However, plasma levels of glucose and hepatic TG were suppressed with isomaltulose supplementation. ${ }^{27}$ Sucrose has been shown to rapidly increase blood glucose level, whereas isomaltulose delays increases in blood glucose and leucrose is slowly degraded into glucose and fructose by $\alpha$-glucosidases. ${ }^{22,27}$ Additional studies are needed to elucidate the mechanistic details regarding the effects of leucrose on obesity-related metabolic abnormalities.

The liver is a major site for regulation of glucose and lipid homeostasis. A diet rich in fats or carbohydrates can lead to an accumulation of fat in the liver, and excessive accumulation of TG in the liver is associated with alteration in glucose. ${ }^{1}$ In the present study, leucrose supplementation reduced the accumulation of lipids and TG in the liver by regulating expression of the lipogenic genes, Pparg, Srebfl, and Fasn. PPAR $\gamma$ is predominantly expressed in adipose tissue, and is expressed at higher levels under condition of insulin resistance and fatty liver. Fat accumulation in liver has been linked to impaired $\beta$-oxidation, and inhibition of $\beta$-oxidation can lead to TG accumulation. ${ }^{28}$ PPAR $\gamma$ is a key regulator to maintain lipid homeostasis and its agonist inhibited statosis in obesie mice model. ${ }^{29}$ CPT1A regulates peroxisomal mitochondrial $\beta$-oxidation by inducing transportation of acyl-carnitine to the inner mitochondrial membrane. In the 
present study, leucrose supplementation recovered expression of Cptla which was down-regulated by a HFD. Consistent with this result, Matsuo et al. ${ }^{30}$ reported that 8 weeks of a $55.7 \%$ isomaltulose diet resulted in higher mRNA level of hepatic Cptla and $A \operatorname{cox} 1$ in rats. Taken together, these results suggest that a benefit of alternative sweeteners may be up-regulation of fatty acid $\beta$-oxidation. A possible model would involve suppression of plasma glucose by leucrose, blocking of de novo lipogenesis in the liver, and increased fatty acid oxidation.

In conclusion, the results of this study indicate that leucrose represents a sugar substitute with the potential to reduce hepatic lipid accumulation and improve blood glucose level by regulating hepatic lipogenesis and $\beta$-oxidation. It remains for lecuroserelated signaling mechanisms and the effect of leucrose on other organs, including the effects of leurose on muscle for obesity-related metabolic abnormalities to be investigated in future studies.

\section{ACKNOWLEDGMENTS}

This research was supported by National Research Foundation of Korea (NRF-2015R1A2A1A15056119).

\section{CONFLICTS OF INTEREST}

No potential conflicts of interest were disclosed.

\section{REFERENCES}

1. Fabbrini E, Sullivan S, Klein S. Obesity and nonalcoholic fatty liver disease: biochemical, metabolic, and clinical implications. Hepatology 2010;51:679-89.

2. Poirier P, Giles TD, Bray GA, Hong Y, Stern JS, Pi-Sunyer FX, et al. Obesity and cardiovascular disease: pathophysiology, evaluation, and effect of weight loss. Arterioscler Thromb Vasc Biol 2006;26:968-76.

3. Kawano Y, Cohen DE. Mechanisms of hepatic triglyceride accumulation in non-alcoholic fatty liver disease. J Gastroenterol 2013:48:434-41.

4. Guturu P, Duchini A. Etiopathogenesis of nonalcoholic steatohepatitis: role of obesity, insulin resistance and mechanisms of hepatotoxicity. Int J Hepatol 2012;2012:212865.

5. Li X, Li Y, Yang W, Xiao C, Fu S, Deng Q, et al. SREBP-1c overexpression induces triglycerides accumulation through increasing lipid synthesis and decreasing lipid oxidation and VLDL assembly in bovine hepatocytes. J Steroid Biochem Mol Biol 2014:143:174-82.

6. Li M, Ye T, Wang XX, Li X, Qiang O, Yu T, et al. Effect of octreotide on hepatic steatosis in diet-induced obesity in rats. PLoS One 2016;11:e0152085

7. Inoue $\mathrm{M}$, Ohtake $\mathrm{T}$, Motomura $\mathrm{W}$, Takahashi N, Hosoki $\mathrm{Y}$,
Miyoshi S, et al. Increased expression of PPARgamma in high fat diet-induced liver steatosis in mice. Biochem Biophys Res Commun 2005:336:215-22.

8. Morán-Salvador E, López-Parra M, García-Alonso V, Titos E, Martínez-Clemente M, González-Périz A, et al. Role for PPAR $\gamma$ in obesity-induced hepatic steatosis as determined by hepatocyteand macrophage-specific conditional knockouts. FASEB J 2011; 25:2538-50.

9. Evans RM, Barish GD, Wang YX. PPARs and the complex journey to obesity. Nat Med 2004;10:355-61.

10. Warfel JD, Vandanmagsar B, Dubuisson OS, Hodgeson SM, Elks $\mathrm{CM}$, Ravussin E, et al. Examination of carnitine palmitoyltransferase 1 abundance in white adipose tissue: implications in obesity research. Am J Physiol Regul Integr Comp Physiol 2017;312:R816-20.

11. Varanasi U, Chu R, Chu S, Espinosa R, LeBeau MM, Reddy JK. Isolation of the human peroxisomal acyl-CoA oxidase gene: organization, promoter analysis, and chromosomal localization. Proc Natl Acad Sci U S A 1994:91:3107-11.

12. Vos MB, Lavine JE. Dietary fructose in nonalcoholic fatty liver disease. Hepatology 2013:57:2525-31.

13. Softic $\mathrm{S}$, Cohen DE, Kahn CR. Role of dietary fructose and hepatic de novo lipogenesis in fatty liver disease. Dig Dis Sci 2016;61:1282-93.

14. Malik VS, Popkin BM, Bray GA, Després JP, Hu FB. Sugar-sweetened beverages, obesity, type 2 diabetes mellitus, and cardiovascular disease risk. Circulation 2010;121:1356-64.

15. Kroger M, Meister K, Kava R. Low-calorie sweeteners and other sugar substitutes: a review of the safety issues. Compr Rev Food Sci Food Saf 2006;5:35-47.

16. Pikis A, Immel S, Robrish SA, Thompson J. Metabolism of sucrose and its five isomers by Fusobacterium mortiferum. Microbiology 2002;148:843-52.

17. Jonker D, Lina BA, Kozianowski G. 13-Week oral toxicity study with isomaltulose (Palatinose) in rats. Food Chem Toxicol 2002; 40:1383-9.

18. Park MO, Lee BH, Lim E, Lim JY, Kim Y, Park CS, et al. Enzymatic process for high-yield turanose production and its potential property as an adipogenesis regulator. J Agric Food Chem 2016;64:4758-64.

19. Chung JY, Kim YS, Kim Y, Yoo SH. Regulation of inflammation by sucrose isomer, Turanose, in raw 264.7 cells. J Cancer Prev 2017;22:195-201.

20. Elias PS, Benecke H, Schwengers D. Safety evaluation studies of leucrose. J Am Coll Toxicol 1996;15:205-18.

21. Bailey RW, Bourne EJ. Formation of leucrose in dextran-producing cultures of Streptococcus bovis. Nature 1959;184(Suppl 12): 905-6.

22. Lee BH, Rose DR, Lin AH, Quezada-Calvillo R, Nichols BL, Hamaker BR. Contribution of the individual small intestinal $\alpha$-glucosidases to digestion of unusual $\alpha$-linked glycemic disaccharides. J Agric Food Chem 2016;64:6487-94.

23. Lee D, Lee J, Hong MG, Lee BH, Kim YM, Chang PS, et al. Optimization of leucrose production by dextransucrase from Streptocuccs mutans and its application as an adipogenesis regualtor. J Funct Foods 2017:39:238-44.

24. Norris AW, Chen L, Fisher SJ, Szanto I, Ristow M, Jozsi AC, et al. Muscle-specific PPARgamma-deficient mice develop increased adiposity and insulin resistance but respond to thiazolid- 
inediones. J Clin Invest 2003;112:608-18.

25. Moore JB, Gunn PJ, Fielding BA. The role of dietary sugars and de novo lipogenesis in non-alcoholic fatty liver disease. Nutrients 2014;6:5679-703.

26. Lim SM, Kim E, Shin JH, Seok PR, Jung S, Yoo SH, et al. Xylobiose prevents high-fat diet induced mice obesity by suppressing mesenteric fat deposition and metabolic dysregulation. Molecules 2018:23:E705.

27. Keyhani-Nejad F, Irmler M, Isken F, Wirth EK, Beckers J, Birkenfeld AL, et al. Nutritional strategy to prevent fatty liver and insulin resistance independent of obesity by reducing glucose-dependent insulinotropic polypeptide responses in mice. Diabetologia 2015:58:374-83.
28. Musso G, Gambino R, Cassader M. Recent insights into hepatic lipid metabolism in non-alcoholic fatty liver disease (NAFLD). Prog Lipid Res 2009;48:1-26.

29. Chou CJ, Haluzik M, Gregory C, Dietz KR, Vinson C, Gavrilova O, et al. WY14,643, a peroxisome proliferator-activated receptor alpha (PPARalpha) agonist, improves hepatic and muscle steatosis and reverses insulin resistance in lipoatrophic A-ZIP/F-1 mice. J Biol Chem 2002;277:24484-9.

30. Matsuo K, Arai H, Muto K, Fukaya M, Sato T, Mizuno A, et al. The anti-obesity effect of the palatinose-based formula inslow is likely due to an increase in the hepatic PPAR-alpha and adipocyte PPAR-gamma gene expressions. J Clin Biochem Nutr 2007;40: 234-41. 